\title{
Pseudoepitheliomatous keratotic and micaceous balanitis: low-risk human papilloma virus detection in two further
} cases

International Journal of STD \& AIDS 0 (0) $1-4$

(C) The Author(s) 2020

Article reuse guidelines:

sagepub.com/journals-permissions DOI: $10.1177 / 095646242096 / 947$

journals.sagepub.com/home/std

@SAGE

\author{
Marialuisa Corbeddu', Luca Pilloni², Roberta Satta', \\ Laura Atzori' (D) and Franco Rongioletti'
}

\begin{abstract}
We report two cases of histologically documented pseudoepitheliomatous keratotic and micaceous balanitis in middleaged male patients, which showed positivity for low-risk serotype human papillomavirus DNA. To our knowledge, only one other case has been documented. Further immunohistochemical proliferative markers were performed and compared to literature findings in penile epithelial proliferations. Evolution to invasive verrucous carcinoma has been associated with absence of HPV DNA. Thus, if confirmed by further studies, HPV testing should be included in pseudoepitheliomatous keratotic and micaceous balanitis assessment to address prognosis, and management.
\end{abstract}

\section{Keywords}

Human papilloma virus, pseudoepitheliomatous keratotic and micaceous balanitis, verrucous carcinoma, immunohistochemistry

Date received: II July 2020; accepted: 5 September 2020

\section{Introduction}

Pseudoepitheliomatous keratotic and micaceous balanitis (PKMB) is an acquired condition, firstly described by Lortat-Jacob and Civatte in 1961. ${ }^{1}$ It remains a rare, probably underestimated disorder of unknown aetiology, ${ }^{2-5}$ with controversial potential for malignant progression. ${ }^{6-8}$ Treatment is often variable, ranging from topical therapy with imiquimod, 5fluoruracil, podophyllin, steroids to physical therapies such as cryotherapy or topical photodynamic therapy, electrocoagulation and/or $\mathrm{CO}_{2}$ laser. ${ }^{9,10}$ Advanced stages require wide margins surgery. ${ }^{11}$

Correlation with human papillomavirus infection (HPV) was reported in only one patient to date, ${ }^{12}$ and we discuss two new cases positive for low risk human papillomavirus DNA.

\section{Report of cases}

The first case is an uncircumcised, 65-year-old white man, with a one-year history of a slowly enlarging, asymptomatic whitish plaque on the glans. He had type 2 diabetes and previous surgical procedures for urethral stenosis about 20 years before. At the time of presentation, the whole of the glans penis was covered by a thick, adherent, nail-like whitish pellicle, extending towards the preputial area (Figure 1). Some micalike scales, with free edges, were present especially on the margin of the glans and prepuce, which could be partially peeled off without bleeding. Careful examination of genitalia, perianal and other areas of the body excluded warty lesions. The patient denied a history of previous lesions suggestive of HPV infection. An incisional diagnostic biopsy, followed by surgery with

\footnotetext{
'Department of Medical Sciences and Public Health, Dermatology Clinic, University of Cagliari, Cagliari, Italy

${ }^{2}$ Department of Medical Sciences and Public Health, Section of Pathology, University of Cagliari, Cagliari, Italy
}

\section{Corresponding author:}

Laura Atzori, Clinica Dermatologica, Via Ospedale 54, 09124 Cagliari, Italy.

Email: atzoril@unica.it 

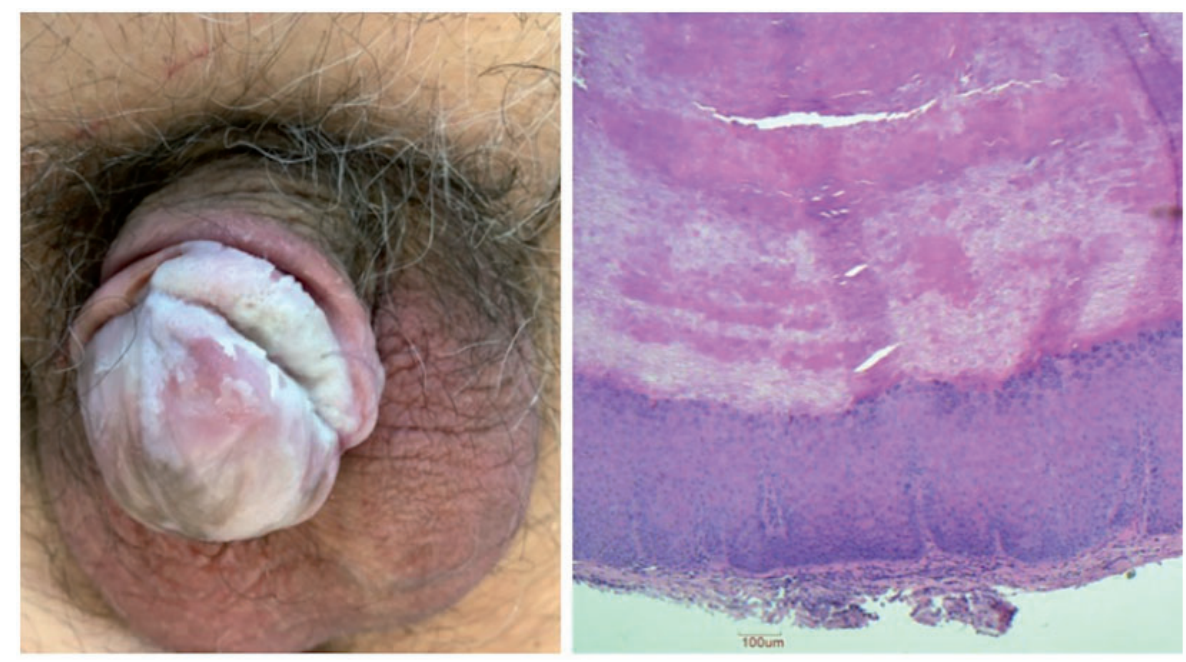

Figure I. Case I: thick whitish, adherent keratotic plaque, of the gland and preputial sheet; histopathologic findings of the shave biopsy showed a widespread pseudoepitheliomatous hyperplasia, acanthosis, hyper-orthokeratosis and elongated rete-ridges, without atypical cells nor koilocytes (Haematoxylin-eosin stain; magnification IOX).

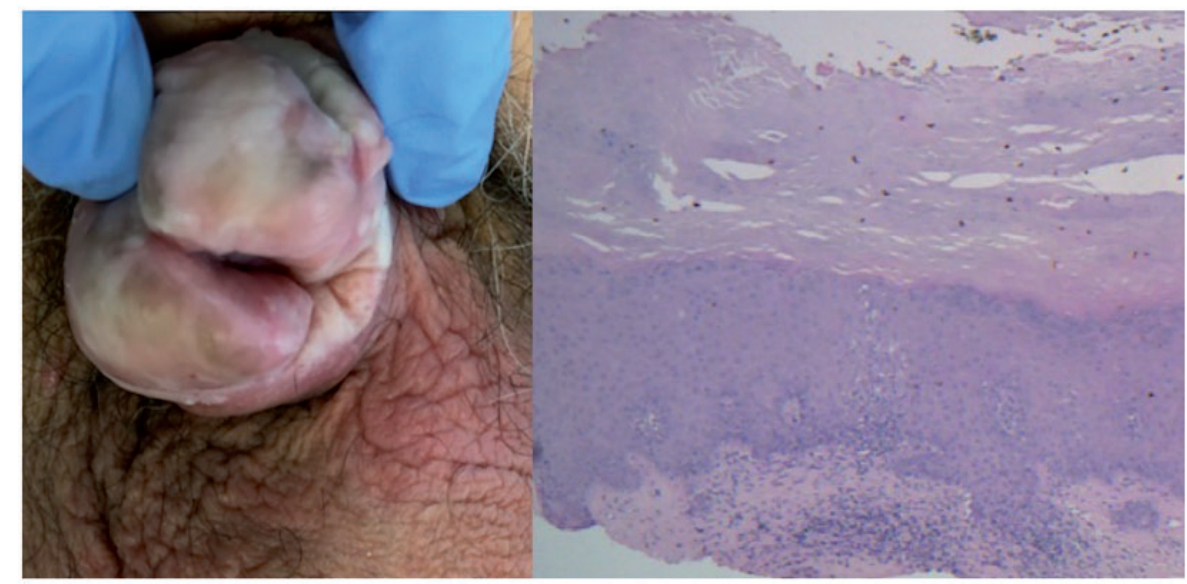

Figure 2. Case 2: White-yellowish keratotic plaque of the balano-preputial sulcus, gland and meatal orifice; histopathologic findings showed hyper-orthokeratosis, acanthosis, and a perivascular non-specific dermal inflammatory infiltrate (Haematoxylin-eosin stain; magnification (OX)).

complete excision was performed, but lesions recurred after few months. Sequential sessions of imiquimod 5\% cream application, cryotherapy with liquid nitrogen and $\mathrm{CO}_{2}$ laser were provided to control relapse. Careful assessment excluded malignant transformation in the 5 year-follow-up.

The second white patient, aged 52 years old, also uncircumcised, presented with a complete whitish to yellow-green induration of the glans and prepuce, of about 3 years' duration (Figure 2). Upon examination the glans skin was hard and inelastic. On the prepuce, more exophytic warty scales could be removed by gently shaving, resembling mica sheets. The patient did not complain of pain or local symptoms, except for some dysparareunia. He was otherwise healthy, and complete body assessment excluded other cutaneous manifestations suggestive of HPV infection or sexually transmitted infections. Routine laboratory work-up was noncontributory. The patient underwent an incisional biopsy for histological examination, followed by a complete surgical excision, without subsequent relapse.

In both cases histopathological examination (Figures 1 and 2) showed pseudoepitheliomatous epidermal hyperplasia and hyper-orthokeratosis along with a superficial dermal infiltrate of lymphocytes, and a few eosinophils and plasma cells. Neither atypical cells nor koilocytes were identified. Immunohistochemical examination was p16 and p53 negative and Ki-67 positive. 
Table I. Immunohistochemical profile of penile lesions compared to described PKMB cases.

\begin{tabular}{lllll}
\hline & Pl6 & P53 & ki67 & HPV \\
\hline Squamous hyperplasia & - & - & $+l-$ & $+l-$ \\
Penile intraepithelial lesions (PeINs) & - & $+l-$ & + & $+l-$ \\
Basaloid and warty PelNs & + & $+l-$ & + & High risk HPV \\
Invasive penile squamous cell carcinoma & +++ & +++ & +++ & High risk HPV \\
Our PKMB cases & - & - & + & Low risk HPV \\
\hline
\end{tabular}

Based on clinical and histopathological features, the diagnosis of plaque stage PKMB was made. The search for human papillomavirus (HPV) DNA, using Digene Hybrid Capture system Kit, showed positivity for lowrisk serotypes ${ }^{6,11}$ in both cases.

\section{Discussion}

PKMB is an uncommon condition, characterized by whitish hyperkeratotic plaques on the glans and balano-preputial sulcus of adult patients. The term micaceous refers to resemblance to mica, a kind of aluminium silicate. ${ }^{2}$ Usually asymptomatic, the condition might be painful and the urethral meatus involvement might cause functional disability. ${ }^{2-5}$ Histopathology shows hyper-orthokeratosis, or parakeratosis, acanthosis, elongated rete ridges, and a dermal inflammatory infiltrate made of eosinophils and lymphocytes. Etiopathogenesis and predisposing factors are unknown. Both our patients were uncircumcised, but the disease occurs also in circumcised patients. ${ }^{3}$ Differential diagnoses include psoriasis, giant condyloma, erythroplasia of Queyrat and verrucous carcinoma. The clinical course is unpredictable, with slow progression and frequent recurrences notwithstanding very extensive surgery. ${ }^{11}$ Progression to verrucous carcinoma is a major concern, and it has been proposed to change the disease name into "micaceous and verrucous malignant balanitis". 7,8 A not unanimously shared classification ${ }^{7}$ suggests an evolution into stages, from the initial plaque stage, to the tumour "nodular" stage, up to the true verrucous carcinoma development, locally invasive with potential lymph node involvement and metastasis. Other than verrucous carcinoma, ${ }^{7,8}$ PKMB has been associated with invasive squamous cell carcinoma ${ }^{1}$ and lethal fibrosarcoma. ${ }^{6}$ Unfortunately, PKMB prognostic factors indicative of a major progression risk are not defined. Different immunohistochemical profiles of penile lesions have been recently proposed, ${ }^{13}$ and we performed p16, p53, Ki-67 assay to characterize PKMB. The comparison is showed in Table 1: squamous hyperplasia is p16 and p53 negative, with variable Ki-67 positivity; differentiated penile intraepithelial lesions (PelNS) are p16 negative, Ki-67 positive, with variable p53 positivity, while basaloid and warty PeINs are p16 and Ki-67 positive, with variable p53 positivity]. Moreover, p16 overexpression, together with p53 and ki67 correlate with high-grade invasive penile tumours, thus differencing them from keratinising lesions with low grade of malignancy. ${ }^{14-16}$

Findings in our two cases were suggestive of simple penile squamous hyperplasia (p16 and p53 negative), with a weak ki67 positivity. Demonstration of HPVDNA in the lesions could be related with Ki-67 expression, as a proliferative marker previously associated with infected HPV cells. ${ }^{16}$ The presence of HPV in PKMB is a controversial issue, documented only in one previous 62-year-old Korean patient. ${ }^{12}$ Another study on PKMB progression into verrucous carcinoma excluded HPV DNA presence and the authors argumed against a role for HPV in the pathogenesis of PKMB. ${ }^{8}$ Our findings reopen the discussion, suggesting a possible association with low-risk serotypes. Presence of the virus does not imply a significant role in PKMB pathogenesis, but it is worthwhile to include HPV DNA exam in the assessment of this uncommon condition, especially if the association with a more benign behaviour ${ }^{17}$ will be confirmed in more large series.

In conclusion, PKMB is a challenging penile condition, with unsolved questions regarding pathogenesis, prognosis and treatment. Reporting is recommended to assess the effective risk of evolution towards malignant and sometimes lethal conditions.

\section{Authors' contribution}

The authors certify that the manuscript is original and never submitted to an other journal for publication before. All authors contributed equally to the manuscript concept and design, as well as to revision and approval of the final text.

\section{Declaration of conflicting interests}

The author(s) declared no potential conflicts of interest with respect to the research, authorship, and/or publication of this article.

\section{Funding}

The author(s) received no financial support for the research, authorship, and/or publication of this article. 


\section{ORCID iD}

Laura Atzori (D) https://orcid.org/0000-0002-8105-1402

\section{References}

1. Lortat-Jacob E and Civatte J. Micaceous and keratosic pseudo-epitheliomatous balanitis. Bull Soc Fr Dermatol Syphiligr 1961; 68: 164-167.

2. Zawar V, Kirloskar M and Chuh A. Watering-can penis' in pseudoepitheliomatous, keratotic and micaceous balanitis. Acta Derm Venereol 2004; 84: 329-330.

3. Perry D, Lynch PL and Fazel N. Pseudoepitheliomatous, keratotic, and micaceous balanitis: case report and review of the literature. Dermatol Nurs 2008; 20: 117-120.

4. Krunic AL, Djerdj K, Starcevic-Bozovic A, et al. Pseudoepitheliomatous, keratotic and micaceous balanitis. Case report and review of the literature. Urol Int 1996; 56: 125-128.

5. Jenkins D and Jakubovic HR. Pseudoepitheliomatous, keratotic, micaceous balanitis. J Am Acad Dermatol 1988; 18: 419-422.

6. Irvine C, Anderson JR and Pye RJ. Micaceous and keratotic pseudoepitheliomatous balanitis and rapidly fatal fibrosarcoma of the penis occurring in the same patient. Br J Dermatol 1987; 116: 719-725.

7. Beljaards RC, Van Dijk E and Hausman R. Is pseudoepitheliomatous, micaceous and keratotic balanitis synonymous with verrucous carcinoma? Br J Dermatol 1987; 117: 641-646.

8. Child FJ, Kim BK, Ganesan R, et al. Verrucous carcinoma arising in pseudoepitheliomatous keratotic and micaceous balanitis, without evidence of human papillomavirus. Br J Dermatol 2000; 143: 183-187.

9. Choo KJL, Ng SK, Sim CS, et al. Pseudoepitheliomatous keratotic and micaceous balanitis treated with topical 5- fluorouracil and liquid nitrogen. Clin Exp Dermatol 2017; 42: 424-426.

10. Zhu H, Jiang Y, Watts M, et al. Treatment of pseudoepitheliomatous, keratotic, and micaceous balanitis with topical photodynamic therapy. Int J Dermatol 2015; 54: 245-247.

11. Kim JY, Kim JY, Park M, et al. Surgical managements of pseudoepitheliomatous keratotic and micaceous balanitis: a case report. Int J Surg Case Rep 2019; 55: 37-40.

12. Kang BS, Lee SD, Park YM, et al. Is recurrent pseudoepitheliomatous keratotic balanitis related to human papillomavirus infection? Acta Derm Venereol 2010; 90: 208-209.

13. Chaux A, Pfannl R, Rodríguez IM, et al. Distinctive immunohistochemical profile of penile intraepithelial lesions: a study of 74 cases. Am J Surg Pathol 2011; 35: 553-562.

14. Cubilla AL, Lloveras B, Alejo M, et al. Value of p16INK4a in the pathology of invasive penile squamous cell carcinomas: a report of 202 cases. Am J Surg Pathol 2011; 35: 253-261.

15. Poetsch M, Hemmerich M, Kakies C, et al. Alterations in the tumor suppressor gene p16(INK4A) are associated with aggressive behavior of penile carcinomas. Virchows Arch 2011; 458: 221-229.

16. Protzel C, Knoedel J, Zimmermann U, et al. Expression of proliferation marker Ki67 correlates to occurrence of metastasis and prognosis, histological subtypes and HPV DNA detection in penile carcinomas. Histol Histopathol 2007; 22: 1197-1204.

17. Cai T, Di Vico T, Durante J, et al. Human papilloma virus and genitourinary cancers: a narrative review. Minerva Urol Nefrol 2018; 70: 579-587. 